\title{
Insights into the complex world of amorphous solids
}

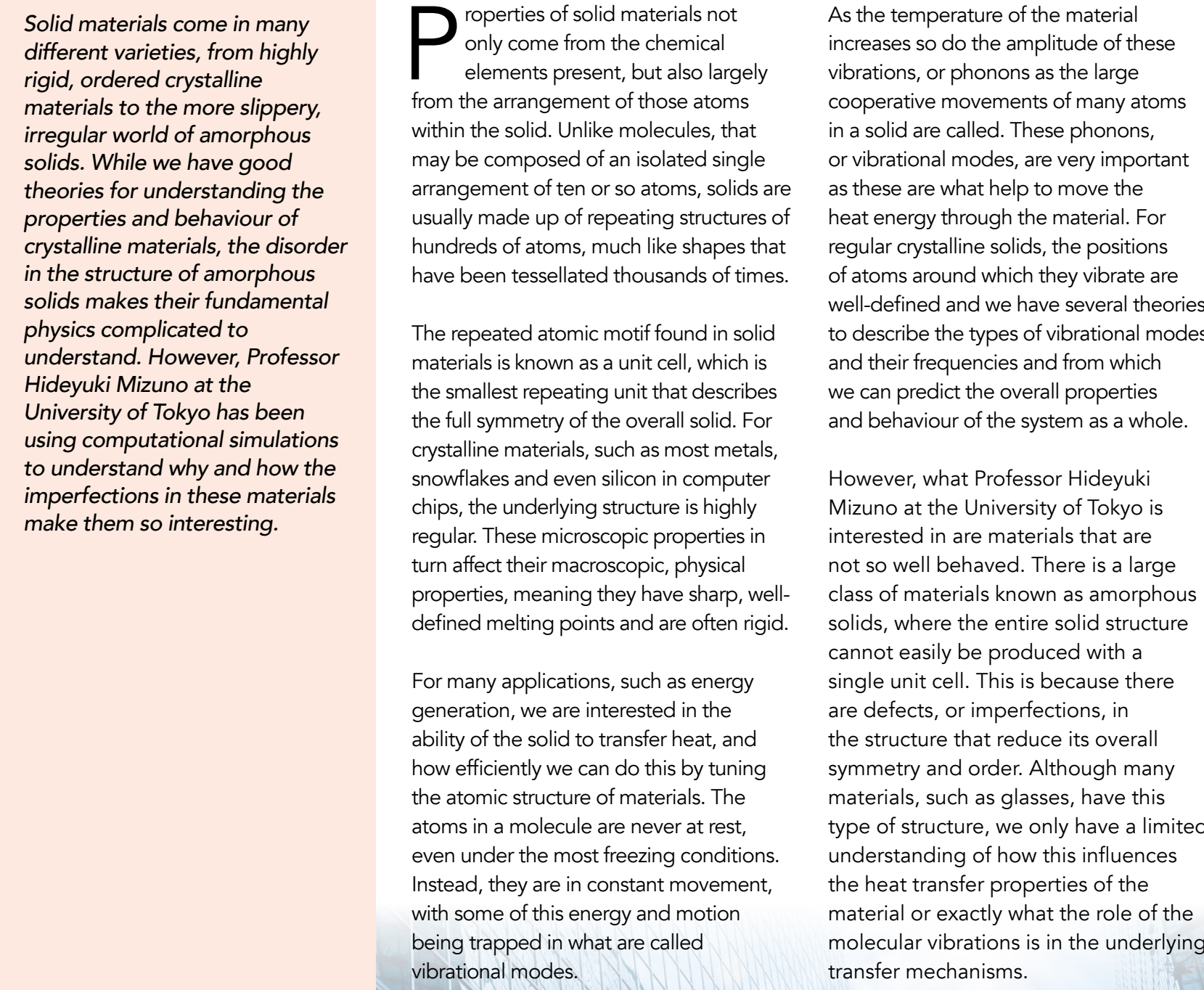

roperties of solid materials not elements present, but also largely from the arrangement of those atoms within the solid. Unlike molecules, that may be composed of an isolated single arrangement of ten or so atoms, solids are usually made up of repeating structures of hundeds of atoms,

The repeated atomic motif found in solid materials is known as a unit cell, which is the smallest repeating unit that describes the full symmetry of the overall solid. For crystalline materials, such as most metals snowflakes and even silicon in computer chips, the underlying structure is highly regular. These microscopic properties in turn affect their macroscopic, physical properties, meaning they have sharp, well-
defined melting points and are often rigid. For many applications, such as energy generation, we are interested in the ability of the solid to transfer heat, and how efficiently we can do this by tuning the atomic structure of materials. The even inder the most freezing conditions. Instead, they are in constant movement with some of this energy and motion being trapped in what are called vibrational modes.

As the temperature of the material increases so do the amplitude of these vibrations, or phonons as the large cooperative movements of many atoms in a solid are called. These phonons, or vibrational modes, are very important as these are what help to move the heat energy through the material. For gular crystallin solids, the positions well-defined and we have several theories to describe the types of vibrational modes and their frequencies and from which we can predict the overall properties and behaviour of the system as a whole.

However, what Professor Hideyuki Mizuno at the University of Tokyo is interested in are materials that are not so well behaved. There is a large class of materials known as amorphous solids, where the entire solid structure cannot easily be produced with a single unit cell. This is because there are defects, or imperfections, in the structure that reduce its overall symmetry and order. Although many materials, such as glasses, have this type of structure, we only have a linited the heat transfer properties of the material or exactly what the role of the molecular vibrations is in the underlying transfer mechanisms.

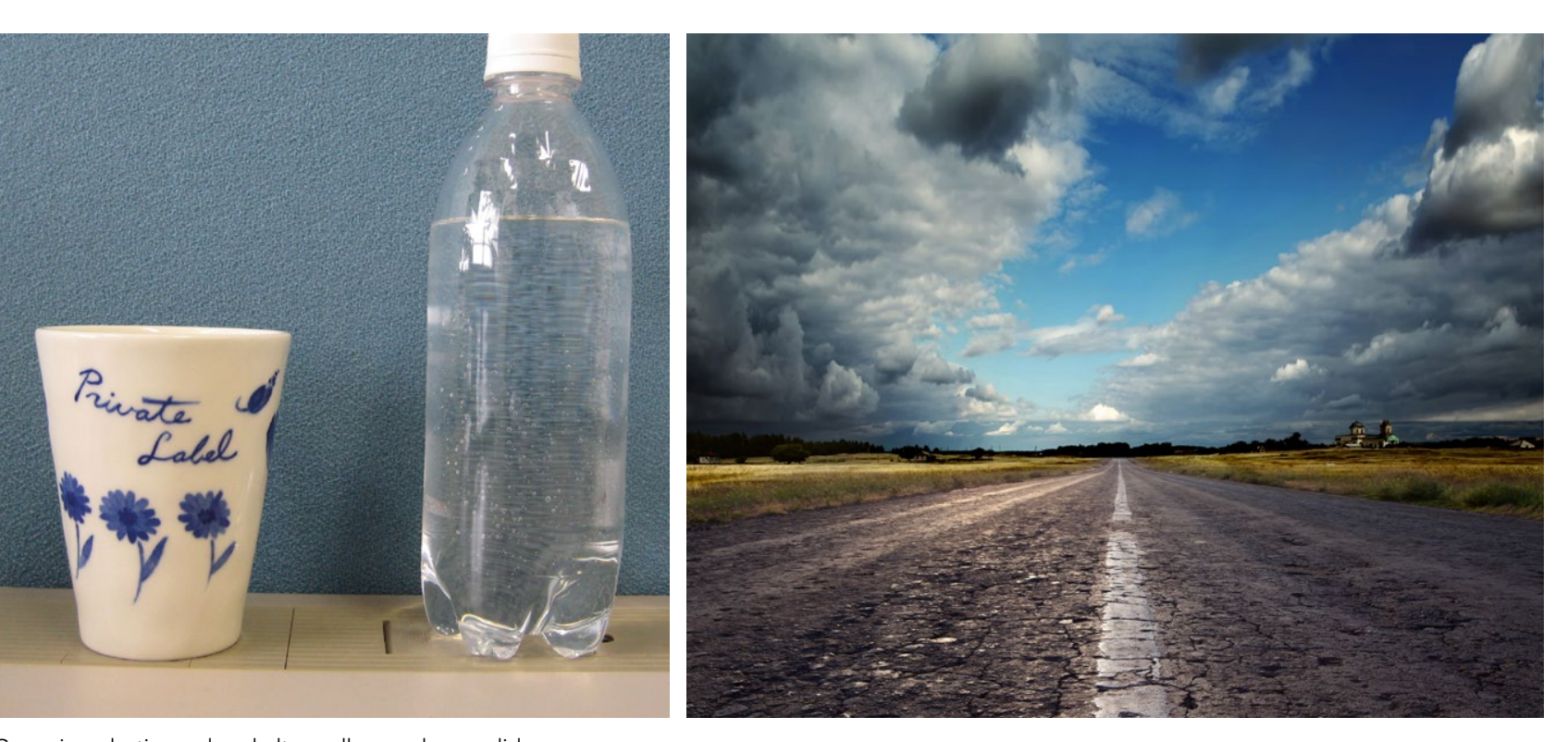

\section{AMORPHOUS DESCRIPTIONS} While glasses are the typical example of such also have the underlying structurat irregularities characteristic of these types of materials. These defects, or structural inhomogeneities, can be exploited to change their physical properties such as elastic stiffness and heat conductance.

Professor Mizuno and his team at the University of Tokyo are experts in creating computational models to describe these complex systems that can be used to explore their underlying physics. In such molecular dynamics simulations, each atom is described as a small sphere with a given charge which can interact with the of modeling the for a sonthe a way exert on each other.

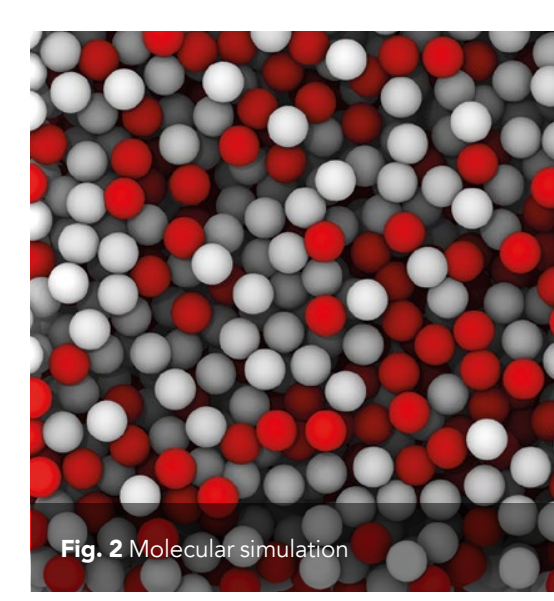

This makes it possible to watch the atoms dance as energy is transferred through the structure.

A particular temperature and amoun of energy is included in the system as the beginning of the simulation By calculating different properties at different time steps, it is possible to visualise the transfer of energy throughout the solid and even follow the movement of all the atoms, making it possible to watch the atoms dance as energy passes through the structu by exciting the vibrational modes.

Molecular dynamics simulations are an systems, that cann toor these

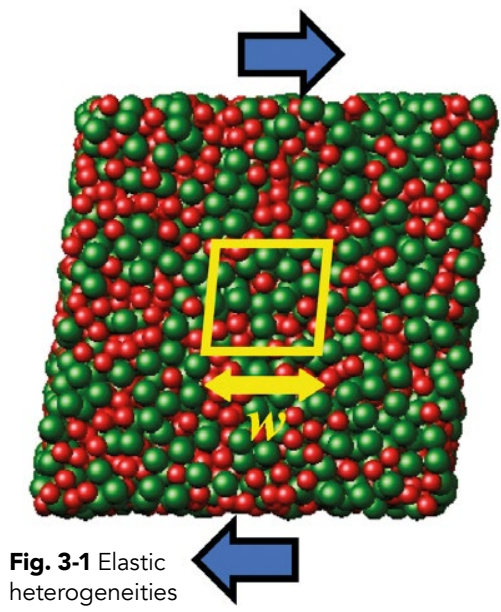

analytical mathematic calculations due to their randomness. They also make it relatively easy to explore 'chemistry on the computer', where atoms can be density of atoms can be increased, and by running the simulation, we can see how this affects the overall properties of the material. This is why molecular dynamics simulations are popular not just in materials design but also as a too pharmaceutical industry.

What is special about the types of simulations that Professor Mizuno does

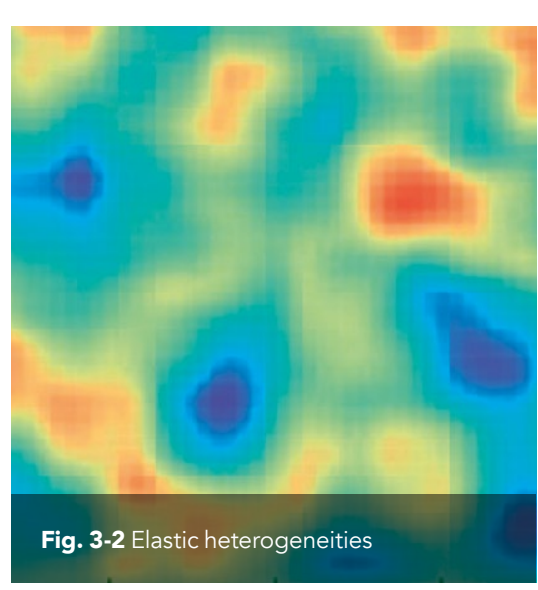
Glasses are the typicade examp
of an ammerphous selid. 
The numerical model
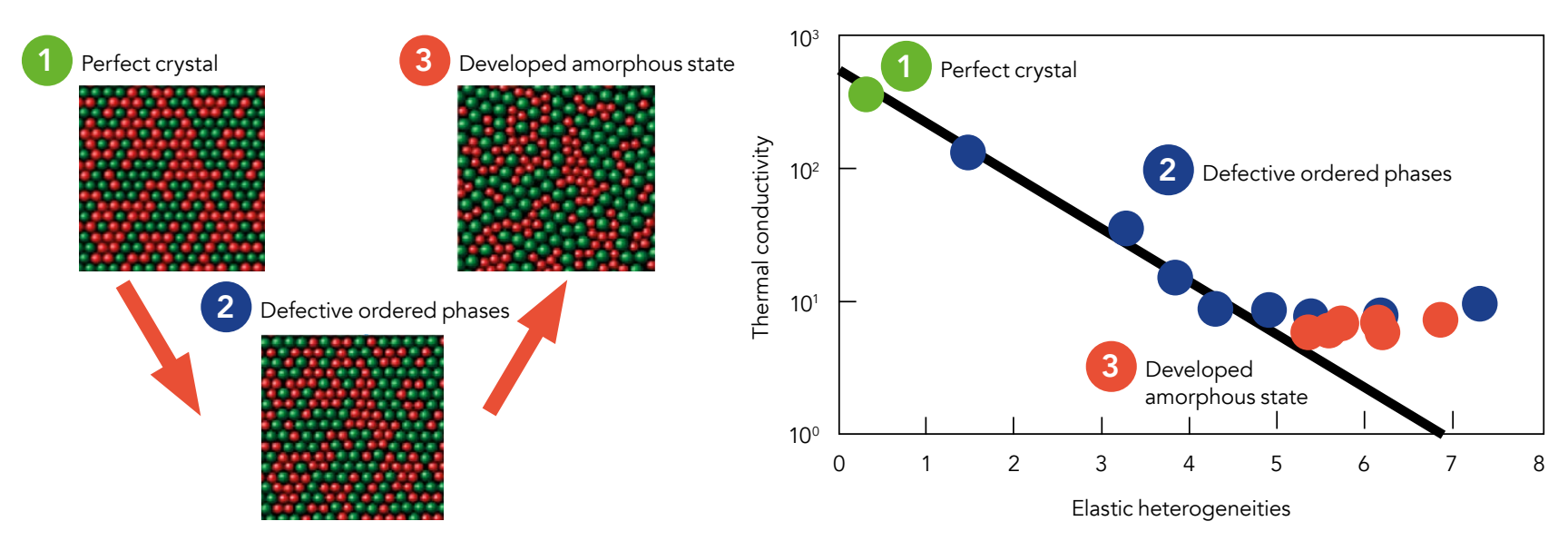

is that he is able to use large-scale numerical simulations to look at the underlying physics in the material. First, he has to create an accurate description of the system, antersiof the number can look at how the low frequency modes of the system behave by manipulating or applying stresses to the system, in the process finding new ways to mode and describe the physics of these low frequency modes in amorphous systems.

\section{VIBRATIONAL TRANSPORT} In his simulations, Professor Mizuno has

This work has found new ways to model and describe the physics of these low frequency modes in amorphous systems. been painstakingly investigating how the material response and properties and type of heterogeneities in the crystalline systems to defect-filled the complexity of the types of motion amorphous ones. By a rigorous analysis of the vibrational modes, and thermal conductivity, Professor Mizuno discerned that properties of
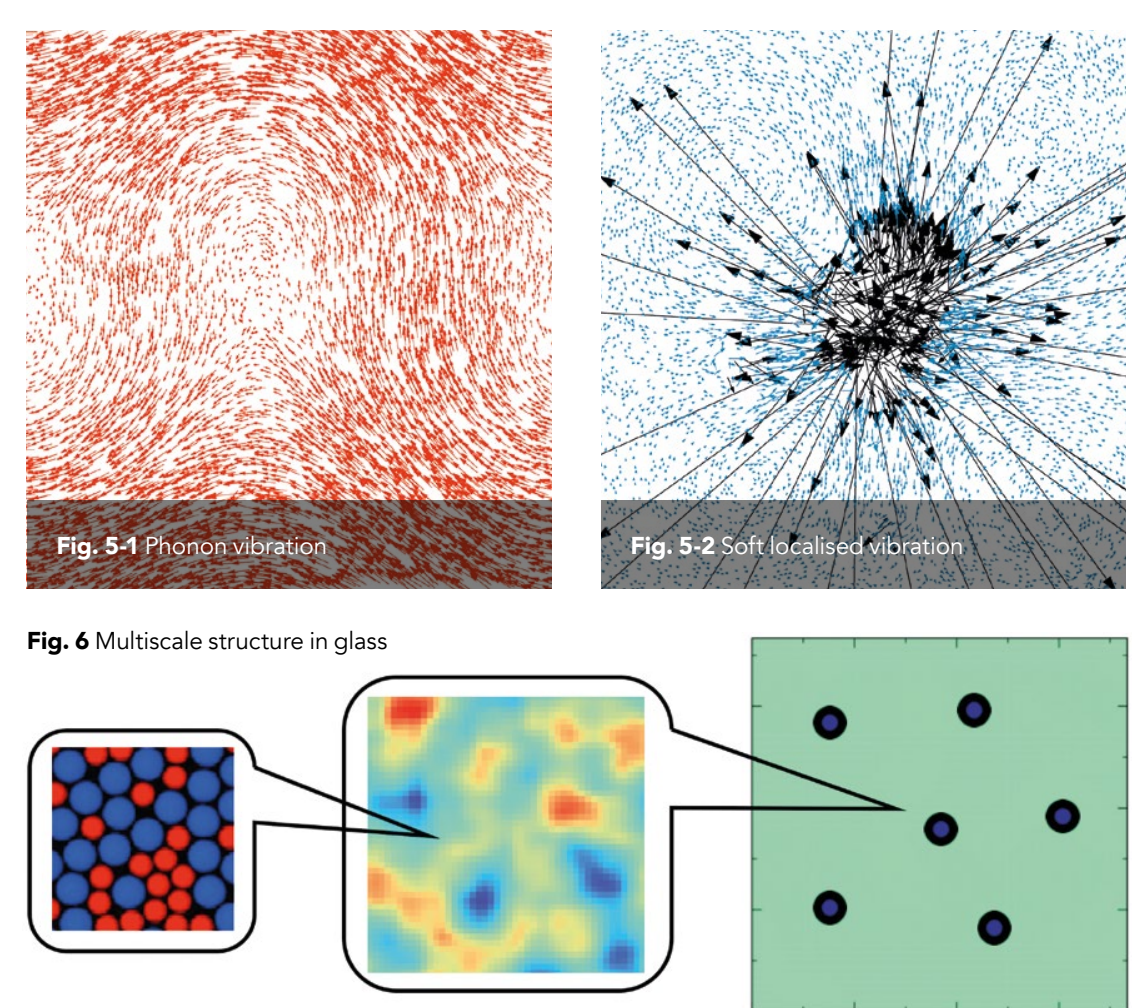
Professor Mizuno argues that amorphous solids are multi-scale structures that behave differently
different scales. structural inhomogeneities in findings provide us with new tools that can be applied to many amorphous

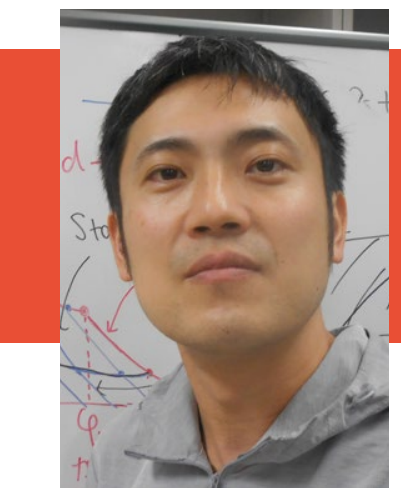

\section{Behind the Research}

\section{Professor Hideyuki Mizuno}

E: hideyukimizuno@phys.c.u-tokyo.ac.jp T: +81354544376 W: http://www.c.u-tokyo.ac.jp/index.html W: http://www.dbs.c.u-tokyo.ac.jp/index.html

Research Objectives

Professor Mizuno's work aims to better understand nonProfessor Mzalline, amorphous solids and to develop solid-staphysics of amorphous materials.

\section{Detail}

Hideyuki Mizuno

Graduate School of Arts and Sciences

The University of Tokyo

Komaba 3-8-1

Tokyo 153-8902

Japan associated with the vibrations in the system, which also makes them more localised to specific areas of the solid.

Somewhat surprisingly, he also found that, for the amorphous systems, there are heterogeneities which are not restricted to local sites but which show the signature of the localised defect vibrations even at limit) Suscopic scale (the continum Iit). Such defect vibrations, which ere specific to the amo nhe systems systems. They disturb the energy transfer in the materials and reduce the heat conductivity.

Professor Mizuno's work represents a huge development in our understanding of how the local amorphous systems do actually affect the overall properties of the materia. they behave differently in different scales and form a 'multi-scale structure' in the amorphous systems. These systems, such as silicate glasses, metallic glasses, plastics, ceramics and asphalts that are widely used

\section{東京大学}

Bio

Hideyuki Mizuno is an Assistant Professor of Graduate School of Arts and Sciences at The University of Tokyo, since 2016. He earned a PhD in Engineering from Kyoto University, Japan, in 2012. He was a postdoctoral fellow in Universie Grin 2012 . He was a postdoctora fellow

\section{Funding}

- Japan Society for the Promotion of Science (JSPS)

\section{Collaborators}

- Jean-Louis Barrat, Université Grenoble Alpes,

Grenoble, France

- Stefano Mossa, Université Grenoble Alpes,

Grenoble, France

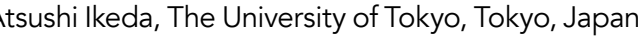

nano SCIENCES

sous légide de la Fondation
Université Grenoble Alpes
References

- Mizuno, H., \& keda, A. (2018). Phonon transport and vibrational excitations in amorphous solids. Physical Review $E_{\text {, }}$ 98(6), 062612. https:///doi.org/10.1103/PhysRevE.98.062612 - Mizuno, H., Shiba, H., \& keda, A. (2017). Continuum limit of the vibrational properties of amorphous solids. Proceeding of the National Academy of Sciences of the United States of America, 114(46), E9767-E9774. https://www.pnas.org/
content/114/46/E9767 - Mizuno, H Mossa, S \& . Relation of vibrational excitations and thermal conductivity to elastic 94(14), 144303. https://doi.org/10.1103/PhysRevB.94.144303 - Mizuno, H., Mossa, S., \& Barrat, J.-L. (2014). Acoustic excitations and elastic heterogeneities in disordered solids. Proceedings of the National Academy of Sciences of the United States of America, 111(33), 11949-11954. https: unww.pnas.org/content/111/33/11949

- Mizuno, H., Mossa, S., \& Barrat, J.-L. (2013). Elastic heterogeneity, vibrational states, and thermal conductivity across an amorphisation transition. Europhysics Letters (EPL), 104(5), 56001. https://iopscience.iop.org/ article/10.1209/0295-5075/104/56001/meta - Mizuno, H., Mossa, S., \& Barrat, J.L. (2013). Measuring spatial distribution of the local elastic modulus in glasses. PhysReve 87.042306 PhysRevE. 87.042306

\section{Personal Response}

What is the greatest challenge in creating these types of simulations for amorphous materials?

II For crystalline materials, we can focus on a unit cell. thanks to their periodicity and symmetry. However, for amorphous materials, we cannot get such benefits and we need to consider the whole system and simulate all the constituent particles. Particularly, in order to study the lower frequency vibrations, we need to analyse the larger system. Up to now, we have accomplished this task on "packing type of glasses' where the interparticle potential is relatively short-ranged. However, we need more efforts for 'network type of glasses such as silicate glasses where we handle the long-range potential, the Coulomb potential. 